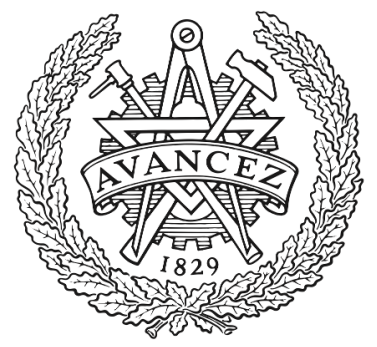

CHALMERS

UNIVERSITY OF TECHNOLOGY

\title{
Low-frequency Noise Characterization of Graphene FET THz Detectors
}

Downloaded from: https://research.chalmers.se, 2023-04-26 10:39 UTC

Citation for the original published paper (version of record):

Yang, X., Vorobiev, A., Jeppson, K. et al (2018). Low-frequency Noise Characterization of Graphene FET THz Detectors. International Conference on Infrared, Millimeter, and Terahertz Waves, IRMMW-THz. http://dx.doi.org/10.1109/IRMMW-THz.2018.8510404

N.B. When citing this work, cite the original published paper. 


\title{
Low-frequency Noise Characterization of Graphene FET THz Detectors
}

\author{
Xinxin Yang ${ }^{1}$, Andrei Vorobiev ${ }^{1}$, Kjell Jeppson $^{1}$, Jan Stake$^{1}$, Luca Banszerus ${ }^{2}$, Christoph Stampfer ${ }^{2}$, \\ Martin Otto ${ }^{3}$ and Daniel Neumaier ${ }^{3}$ \\ ${ }^{1}$ Chalmers University of Technology, SE-41296 Gothenburg, Sweden \\ ${ }^{2}$ RWTH Aachen University, DE-52074 Aachen, Germany \\ ${ }^{3} \mathrm{AMO}$ GmbH, DE-52074 Aachen, Germany
}

\begin{abstract}
Graphene field-effect transistors are promising for direct detection of $\mathrm{THz}$ signals at room temperature. The sensitivity of such detectors can be in part limited by the low-frequency noise. Here, we report on the characterization of the low-frequency noise of graphene field-effect transistor $\mathrm{THz}$ detectors in the frequency range from $1 \mathrm{~Hz}$ to $1 \mathrm{MHz}$. The room-temperature Hooge parameter is extracted to be around $2 \times 10^{-3}$. The voltage responsivity at room-temperature and the corresponding minimum noise equivalent power at $0.3 \mathrm{THz}$ are estimated to be $11 \mathrm{~V} / \mathrm{W}$ and $0.2 \mathrm{nW} / \mathrm{Hz}^{0.5}$, respectively, at a modulation frequency of $333 \mathrm{~Hz}$, which shows comparable results with other detector technologies.
\end{abstract}

\section{INTRODUCTION}

G RAPHENE, with its high carrier mobility and saturation velocity, is a promising material for high-frequency devices. In recent years, room-temperature $\mathrm{THz}$ detectors based on graphene field-effect transistors (GFETs) have been demonstrated [1, 2]. An important figure of merit of direct power detectors is the noise equivalent power (NEP), which corresponds to the lowest detectable power [3]. Most of previous studies have estimated the NEP of GFET THz detectors based on responsivity measurements and thermal noise calculations. Assuming a short integration time, the low-frequency noise can be avoided. However, impurities and other defects introduced during the fabrication process are expected to contribute to the low-frequency noise $[4,5]$, and thereby degrade the detector performance. It is important to understand the noise spectrum for detector applications. In this work, we have characterized the low-frequency noise in GFET $\mathrm{THz}$ detectors. This allows us to find a low frequency limit of the modulation frequency, above which the low-frequency noise is negligible.

\section{RESULTS}

GFET $\mathrm{THz}$ detectors were fabricated using monolayer CVD graphene made by RWTH and AMO as in [2]. Fig. 1 shows an optical micrograph and a schematic cross-sectional view of a GFET THz detector with a mesa length $(L)$ of $0.7 \mu \mathrm{m}$, a gate length of $0.5 \mu \mathrm{m}$ and an effective channel width $(W)$ of
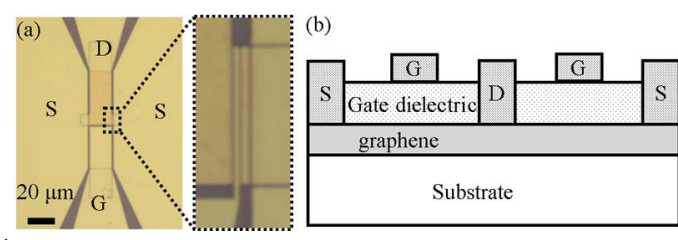

Fig. 1. Optical micrograph (a) and schematic cross-sectional view (b) of a GFET $\mathrm{THz}$ detector with a gate length of $0.5 \mu \mathrm{m}$, and an effective channel width of $20 \mu \mathrm{m}$ (two $10-\mu \mathrm{m}$ wide gates in parallel).
$20 \mu \mathrm{m}$ (two $10-\mu \mathrm{m}$ wide gates in parallel). The rectified $\mathrm{THz}$ voltage $\left(V_{\mathrm{THz}}\right)$ was measured on-wafer using Cascade T-Wave ground-signal-ground probes and an SR830 lock-in amplifier. The 0.3-THz input signal was modulated at $333 \mathrm{~Hz}$. The THz source was composed of a WR-3.4 extender driven by an Agilent $8275 \mathrm{D}$ signal generator. The input $\mathrm{THz}$ power was around $80 \mu \mathrm{W}$ considering of the insertion loss of the probes and the return loss of the detectors. The voltage noise spectral density $\left(S_{\mathrm{V}, 10 \mathrm{mV}}\right)$ of the low-frequency noise was measured at room temperature using a Keysight E4727A Advanced Low-Frequency Noise Analyzer. During the experiment, the sample was placed inside a grounded metal box to minimize environmental noise. The drain-source voltage $\left(V_{\mathrm{DS}}\right)$ was set to $10 \mathrm{mV}$ since limitations in the setup sensitivity make it hard to measure the noise level with the same drain bias as low as $V_{\text {THz }}$. The gate leakage current was less than $0.6 \mathrm{nA}$ posing only negligible effects on the noise level.

Fig. 2 (a) shows the measured drain-source resistance $\left(r_{D S}\right)$ of a typical GFET THz detector plotted versus the gate voltage $\left(V_{\mathrm{GS}}\right)$. By fitting the measured resistance to the model described in [6], the electron mobility, the residual carrier concentration, and the contact resistance were extracted to be $2000 \mathrm{~cm}^{2} / \mathrm{Vs}$, $1.7 \times 10^{12} \mathrm{~cm}^{-2}$, and $22 \Omega$, respectively. The charge carrier concentration $(n)$ was estimated to be in the range from $1.7 \times 10^{12}$ to $5.5 \times 10^{12} \mathrm{~cm}^{-2}$. Fig. 2 (b) shows the rectified $\mathrm{THz}$ voltage as a function of $V_{\mathrm{GS}}$. The rectified $\mathrm{THz}$ voltage is in the range from 0.04 to $0.16 \mathrm{mV}$.
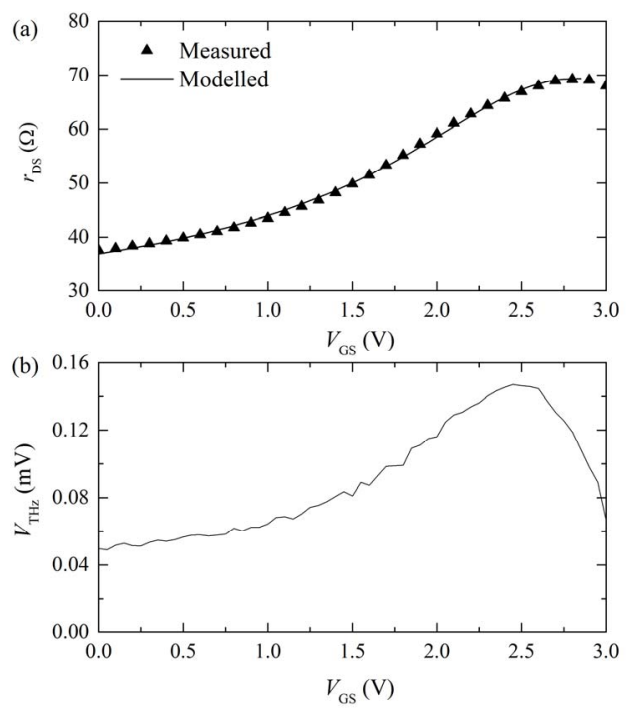

Fig. 2. (a) The measured drain-source resistance of the detector as a function of $V_{\mathrm{GS}}$ (symbols), together with the fitting result (solid line). (b) The measured rectified $\mathrm{THz}$ voltage of the detector as a function of $V_{\mathrm{GS}}$ without drain-source bias at $0.3 \mathrm{THz}$. 

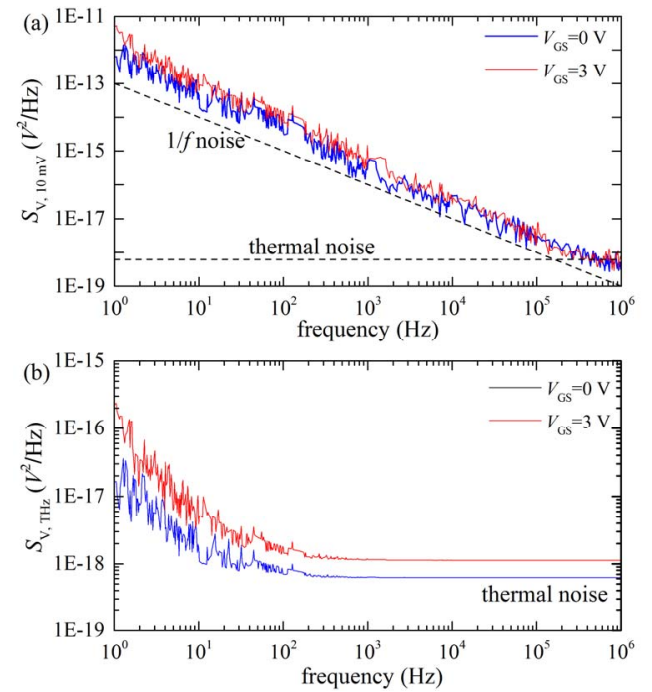

Fig. 3. (a) Measured noise spectral density of the detector vs. $V_{\mathrm{GS}}$ at $V_{D S}=10 \mathrm{mV}$. (b) Calculated noise spectral density of the detector vs. $V_{\mathrm{GS}}$ at $V_{D S}=V_{\mathrm{THz}}$.

Fig. 3 (a) shows the noise spectral density of the detector measured at two different gate voltages together with the thermal noise calculated as in [1]. It can be seen that the noise spectral density reveals a $1 / f$ dependence. The observed $1 / f$ noise is caused by low-frequency fluctuations of the channel resistance due to fluctuations in carrier concentration and/or mobility $[4,5]$. The $1 / f$ noise $\left(\mathrm{S}_{V, 1 / f}\right)$ at a $10 \mathrm{mV}$ drain-source voltage can be estimated by subtracting the calculated thermal noise. Assuming negligible current-induced heating effects, the Hooge model [7] can be used to estimate the $1 / f$ noise of the detector with a drain-source bias at $V_{\mathrm{THz}}$ from,

$$
S_{V \mathrm{THz}, 1 / f}=S_{V, 1 / f}\left(\frac{V_{\mathrm{THz}}}{V_{\mathrm{DS}}}\right)^{2},
$$

where $V_{D S}=10 \mathrm{mV}$. The noise spectral density $\left(S_{V, \mathrm{THz}}\right)$ at $V_{\mathrm{THZ}}$ can now be obtained by again adding the previously subtracted thermal noise as shown in Fig 3 (b). As shown by the graph, the total noise is defined mainly by the thermal noise at frequencies higher than $100 \mathrm{~Hz}$. This means that we can eliminate the effects of the $1 / f$ noise by using modulation frequencies higher than $100 \mathrm{~Hz}$.

We can evaluate the Hooge parameter based on $\mathrm{S}_{V, 1 / f}$ and the device parameters [7]

$$
\alpha_{\mathrm{H}}=\frac{s_{V, 1 / f}}{V_{\mathrm{DS}}^{2}} * N f \approx 2 \times 10^{-3},
$$

where $N=n W L$ is the number of carriers. $\alpha_{\mathrm{H}}$ is $2 \sim 3$ orders of magnitude larger than those of mature semiconductors [7].

Fig. 4 shows the room-temperature $\mathrm{THz}$ voltage responsivity $\left(R_{\mathrm{V}}\right)$ and the NEP, which are important figures of merit for the direct power detector. The voltage responsivity was calculated as in [2], and the NEP was obtained as NEP = $\sqrt{S_{V, \mathrm{THz}}} / R_{V}$. The maximum $R_{\mathrm{V}}$ is $11 \mathrm{~V} / \mathrm{W}$ at $V_{\mathrm{GS}}=2.5 \mathrm{~V}$, and the corresponding minimum NEP is $0.2 \mathrm{nW} / \mathrm{Hz}^{0.5}$. The NEP is at the same level as other detector technologies, e.g. 0.65 $\mathrm{nW} / \mathrm{Hz}^{0.5}$ for silicon MOSFETs [8], $0.4 \mathrm{nW} / \mathrm{Hz}^{0.5}$ for InP DHBTs [9] and $0.1 \mathrm{nW} / \mathrm{Hz}^{0.5}$ for AlGaN/GaN HEMTs [10].

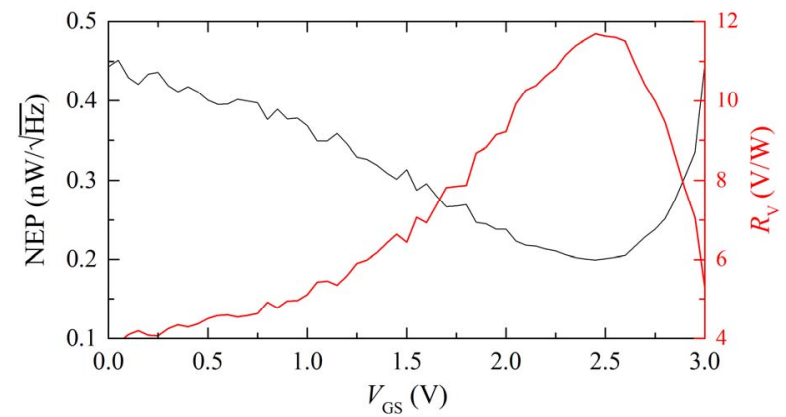

Fig. 4. NEP and voltage responsivity of the detector as a function of $V_{\mathrm{GS}}$ with a signal frequency of $0.3 \mathrm{THz}$.

\section{SUMMARY}

We have characterized the low-frequency noise of GFET $\mathrm{THz}$ detectors and found that modulation frequencies should be higher than $100 \mathrm{~Hz}$ to eliminate the effects of the $1 / f$ noise. In this work, the Hooge parameter is around $2 \times 10^{-3}$, which is $2 \sim 3$ orders of magnitude larger than those of mature semiconductors. The room-temperature responsivity and the corresponding minimum NEP at $0.3 \mathrm{THz}$ were estimated to be $11 \mathrm{~V} / \mathrm{W}$ and $0.2 \mathrm{nW} / \mathrm{Hz}^{0.5}$, respectively. These values show the potential of GFET THz detector to compete with other detector technologies.

\section{ACKNOWLEDGMENT}

This work was supported in part by the EU Graphene Flagship Core 1 Project and in part by the Swedish Research Council (VR) under Grant No. 2017-04504.

\section{REFERENCES}

[1] L. Vicarelli et al., "Graphene field-effect transistors as room-temperature terahertz detectors," Nat. Mater., vol. 11, no. 10, pp. 865-871, 2012.

[2] A. A. Generalov et al., "A 400-GHz graphene FET detector," IEEE Trans. THz Sci. Technol., vol., vol. 7, no. 5, pp. 614-616, 2017.

[3] S. van Berkel et al., "THz imaging using uncooled wideband direct detection focal plane arrays," IEEE Trans. THz Sci. Technol., vol. 7, no. 5, pp. 481-492, 2017.

[4] A. A. Balandin, "Low-frequency $1 / f$ noise in graphene devices," Nat. Nanotechnol., vol. 8, no. 8, pp. 549-555, 2013.

[5] A. Kaverzin, et al., "Impurities as a source of $1 / f$ noise in graphene," Phys. Rev. B, vol. 85, no. 7, p. 075435, 2012.

[6] S. Kim et al., "Realization of a high mobility dual-gated graphene field-effect transistor with $\mathrm{Al}_{2} \mathrm{O}_{3}$ dielectric," Appl. Phys. Lett., vol. 94, no. 6, p. 062107, 2009.

[7] J. Sikula et al., Advanced experimental methods for noise research in nanoscale electronic devices. Springer Science+Business Media, 2006, vol. 151.

[8] K. E. Seery et al., "Characterization of Si-MOSFET CMOS devices for detection at 170 to $250 \mathrm{GHz}$," SPIE OPTO, San Francisco, United States, vol. 105311R, Jan. 27-Feb. 1, 2018.

[9] N. Dyakonova et al., "Reducing noise equivalent power in InP DHBT terahertz detector by biasing the collector," 2017 ICNF, pp. 1-4, Vilnius, Lithuania, Jun. 20-23, 2017.

[10] D. Čibiraitè et al, "Enhanced performance of AlGaN/GaN HEMT-based $\mathrm{THz}$ detectors at room temperature and at low temperature," $201742 \mathrm{nd}$ IRMMW-THz, pp. 1-2, Cancun, Mexico, Aug. 27-Sept. 1, 2017. 